\title{
ROTHE-MARUYAMA DIFFERENCE SCHEME FOR THE STOCHASTIC SCHRÖDINGER EQUATION
}

\author{
Ali Sirma \\ Halic University \\ Beyoglu, Istanbul - 34445, TURKEY
}

\begin{abstract}
In this study, the initial value stochastic Schrödinger type problem in an abstract Hilbert space with the self-adjoint operator is investigated.

Rothe-Maruyama method for the numerical solution of this problem is presented. Theorem on the convergence of this difference scheme is established. A numerical example is given.
\end{abstract}

AMS Subject Classification: 60H30, 35J25, 65H10

Key Words: Rothe-Maruyama differrence scheme; modified Gauss elimination method; stochastic Schrödinger equation

\section{Introduction}

In the literature, stochastic and deterministic type Schrödinger equations have been extensively studied by many researchers (see [2], [3], [6], [11] and the references given therein). Although, in any Hilbert space, numerical approximation of abstract stochastic Schrödinger equation, using Rothe-Maruyama difference scheme has not been studied yet. In this article, the initial value problem for the stochastic Schrödinger equation

$$
i d u(t)+A u(t) d t=f(t) d w_{t}, 0<t<T, u(0)=0
$$

in a Hilbert space $H$ with a self-adjoint positive definite operator $A$ is considered. For the approximate solution of (1), first order of accuracy RotheMaruyama difference scheme is constructed. The results are supported by nu-

Received: November 29, 2020

(C) 2021 Academic Publications 
merical implementation. Throughout the paper:

(i) $w_{t}$ is a standard Wiener process given on the probability space $(\Omega, F, P)$.

(ii) $f(z)$ is an element of the space $M_{w}^{2}\left([0, T], H_{1}\right)$ for any $z \in[0, T]$, where $H_{1}$ is a subspace of $H$.

Here, $M_{w}^{2}([0, T], H)$ denote the space of $H$-valued measurable processes which satisfy :

(a) $\phi(t)$ is $F_{t}$ measurable, a.e. in $t$,

(b) $E \int_{0}^{T}\|\phi(t)\|_{H}^{2} d t<\infty$.

Strong, mild and weak solutions of stochastic differential equations are studied by many researchers, as an example see [5], [10]. In the present paper, following [1] and [4], we study the initial value problem (1) in a Hilbert space.

Our main interest in this study is to construct and investigate the singlestep Rothe-Maruyama difference scheme for the numerical solution problem (1). On the segment $[0, T]$ we consider the uniform grid space

$$
[0, T]_{\tau}=\left\{t_{k}=k \tau, k=0,1, \ldots, N, N \tau=T\right\}
$$

with step size $\tau>0$ and $N$ is an arbitrary but fixed positive integer.

Note that for the self-adjoint operator $A$ in a Hilbert space $H$, linear operator $e^{i t A}$ is bounded and it is a strongly continuous semigroup (see [8], [9]). Also,

$$
\left\|e^{i t A}\right\|_{H \rightarrow H} \leq 1
$$

and

$$
u(t)=-i \int_{0}^{t} e^{i(t-s) A} f(s) d w_{s}
$$

is a unique mild solution of the problem (1) under the assumptions $(i)-(i i)$. 


\section{Rothe-Maruyama Difference Scheme}

First, applying the semigroup property of $e^{i t A}$ and single step difference scheme for solution of problem (1) and replacing $e^{i \tau A}$ by $R=(I-i \tau A)^{-1}$, we can construct the corresponding Rothe-Maruyama difference scheme (see [1])

$$
\left\{\begin{array}{l}
i\left(u_{k}-u_{k-1}\right)+\tau A u_{k}=f\left(t_{k-1}\right) \Delta w_{k}, \\
\Delta w_{k}=w_{k}-w_{k-1}, 1 \leq k \leq N, u_{0}=0
\end{array}\right.
$$

for the numerical solution of problem (1). By induction, we can write

$$
u_{k}=-i \sum_{j=1}^{k} R^{k-j+1} f\left(t_{j-1}\right) \Delta w_{j}
$$

for the solution of the Rothe-Maruyama difference scheme (5). Now we show that Rothe-Maruyama difference scheme (5) for the solution of problem (1) has a convergence of order $1 / 2$. It is possible under stronger assumption than $(i i)$ for $f(t)$ : case without Wiener process. Assume that

$$
\max _{0 \leq t \leq T}\left\|A^{2} f(t)\right\|_{H}+\max _{0 \leq t \leq T}\left\|A f^{\prime}(t)\right\|_{H} \leq M_{4}
$$

Moreover, for this we need some related estimates which is stated in the following lemma.

Lemma 1. Let $A$ be a self-adjoint positive definite operator, then the following estimates hold:

$$
\begin{gathered}
\left\|A^{\alpha} R^{k}\right\|_{H \rightarrow H} \leq \frac{M_{1}}{(\sqrt{k} \tau)^{\alpha}}, 1 \leq k \leq N, 0 \leq \alpha \leq 1, \\
\left\|A^{-\beta}\left(R^{k}-e^{i k \tau A}\right)\right\|_{H \rightarrow H} \leq M_{2}(\sqrt{k} \tau)^{\beta}, 1 \leq k \leq N, 1 \leq \beta \leq 2 .
\end{gathered}
$$

Here the positive constants $M_{1}$ and $M_{2}$ do not depend on $k$ and $\tau$ but depend on $\alpha$ and $\beta$, respectively.

Proof. For $0 \leq \alpha \leq 1$ except the case $\alpha=k=1$ using the spectral representations of self-adjoint operators we have

$$
\left\|A^{\alpha} R^{k}\right\|_{H \rightarrow H} \leq \sup _{-\infty<\mu<\infty} \frac{\left|\mu^{\alpha}\right|}{\left(1+\tau^{2} \mu^{2}\right)^{k / 2}} .
$$


Let $g(\mu)=\frac{\mu^{\alpha}}{\left(1+\tau^{2} \mu^{2}\right)^{k / 2}}$. Then, $g(\mu)$ attains its supremum at $g^{\prime}\left(\mu^{*}\right)=0$, that is for $\left(\mu^{*}\right)^{2}=\frac{\alpha}{(k-\alpha) \tau^{2}}$. The supremum of $g(\mu)$ is

$$
\begin{gathered}
g\left(\mu^{*}\right)=\left(\frac{\alpha}{(k-\alpha) \tau^{2}}\right)^{\alpha / 2}\left(\frac{1}{1+\frac{\alpha}{k-\alpha}}\right)^{k / 2}=\frac{\alpha^{\alpha / 2}}{(\sqrt{k} \tau)^{\alpha}}\left(\frac{k-\alpha}{k}\right)^{(k-\alpha) / 2} \\
\leq \frac{\alpha^{\alpha / 2}}{(\sqrt{k} \tau)^{\alpha}} \leq \frac{M_{1}}{(\sqrt{k} \tau)^{\alpha}}
\end{gathered}
$$

Now let us consider the case $\alpha=k=1$. Using the spectral representation of self-adjoint operators, we get

$$
\|A R\|_{H \rightarrow H} \leq \sup _{\infty<\mu<\infty} \frac{|\mu|}{|1-i \tau \mu|} \leq \frac{1}{\tau} .
$$

Hence the estimate $(8)$ holds. Now let $R(s)=(I-i \tau s A)^{-1}$. Then

$$
\begin{gathered}
\left\|A^{-\beta}\left(R^{k}(s)-e^{i k \tau A}\right)\right\|_{H \rightarrow H} \\
=\left\|A^{-\beta} \int_{0}^{1} \frac{d}{d s}\left(R^{k}(s) e^{i k \tau(1-s) A}\right) d s\right\|_{H \rightarrow H} \\
=\left\|A^{-\beta} \int_{0}^{1} i k \tau A R^{k+1}(s) e^{i k \tau(1-s) A}(i \tau s A) d s\right\|_{H \rightarrow H} \\
\leq k \tau^{2} \int_{0}^{1}\left\|A^{-\beta+2} R^{k+1}(s)\right\|_{H \rightarrow H}\left\|e^{i k \tau(1-s) A}\right\|_{H \rightarrow H} s d s \\
\leq k \tau^{2} \int_{0}^{1} \frac{M_{1}}{(\sqrt{k+1} \tau s)^{2-\beta}} s d s \leq M_{1}(\sqrt{k} \tau)^{\beta} .
\end{gathered}
$$

Hence the estimate (9) holds for some positive constant $M_{1}$ depends on $\beta$, but not depends on $k$ and $\tau$.

Theorem 2. Let $A$ be a self-adjoint positive definite operator and $A \geq$ $\delta I(\delta>0)$. Then, the Rothe-Maruyama difference scheme (5) for the solution of problem (1) has a convergence of order $1 / 2$. That is, the convergence estimate

$$
\max _{0 \leq k \leq N}\left(E\left\|u\left(t_{k}\right)-u_{k}\right\|_{H}^{2}\right)^{1 / 2} \leq M \tau^{1 / 2}
$$

holds. Here the positive constant $M$ does not depend on $\tau$. 
Proof. By (6), we have the formula

$$
u\left(t_{k}\right)-u_{k}=T_{1 k}+T_{2 k}+T_{3 k}
$$

where

$$
\begin{gathered}
\left.T_{1 k}=-i \sum_{j=1}^{k}\left[e^{i(k-j) \tau A}-R^{k-j}\right] \int_{t_{j-1}}^{t_{j}} e^{i\left(t_{j}-s\right) A} f(s) d w_{s}\right], \\
T_{2 k}=-i \sum_{j=1}^{k} R^{k-j}\left[\int_{t_{j-1}}^{t_{j}} e^{i\left(t_{j}-s\right) A} f(s) d w_{s}-e^{i \tau A} f\left(t_{j-1}\right) \Delta w_{j}\right], \\
T_{3 k}=-i \sum_{j=1}^{k} R^{k-j}\left[e^{i \tau A}-R\right] f\left(t_{j-1}\right) \Delta w_{j} .
\end{gathered}
$$

We estimate these three terms separetely. First, let us obtain an estimate for $T_{1 k}$. Using the triangle inequality, inequality (9), Ito isometry and estimate (7), we have

$$
\begin{gathered}
E\left\|T_{1 k}\right\|_{H}^{2} \\
\leq \sum_{j=1}^{k}\left\|A^{-1}\left[e^{i(k-j) \tau A}-R^{k-j}\right]\right\|_{H \rightarrow H}^{2} \int_{t_{j}-1}^{t_{j}}\left\|A e^{i\left(t_{j}-s\right) A} f(s)\right\|_{H}^{2} d s \\
\leq \sum_{j=1}^{k} M_{2}^{2}\left((k-j) \tau^{2}\right) \int_{t_{j-1}}^{t_{j}}\|A f(t)\|_{H}^{2} d s \\
\leq \sum_{j=1}^{k} M_{2}^{2} T \tau \int_{t_{j-1}}^{t_{j}}\|A f(t)\|_{H}^{2} d s \leq M_{2}^{2} T^{2} \tau\left(\max _{0 \leq t \leq T}\|A f(t)\|_{H}\right)^{2} .
\end{gathered}
$$

Hence,

$$
\max _{0 \leq k \leq N}\left(E\left\|T_{2 k}\right\|_{H}^{2}\right)^{1 / 2} \leq M_{2} T \tau^{1 / 2} .
$$

Now let us estimate $T_{2 k}$.

$$
=E\left\|\sum_{j=1}^{k} R^{k-j} \int_{t_{j}-1}^{t_{j}}\left[e^{i\left(t_{j}-s\right) A} f(s) d w_{s}-e^{i \tau A} f\left(t_{j-1}\right) \Delta w_{j}\right]\right\|_{H}^{2}
$$




$$
\begin{aligned}
& \leq \sum_{j=1}^{k}\left\|R^{k-j}\right\|_{H \rightarrow H}^{2} E\left\|\int_{t_{j}-1}^{t_{j}}\left(e^{i\left(t_{j}-s\right) A} f(s)-e^{i \tau A} f\left(t_{j-1}\right)\right) d w_{s}\right\|_{H}^{2} \\
& \leq \sum_{j=1}^{k} E\left\|\int_{t_{j}-1}^{t_{j}} \int_{t_{j}-1}^{s} \frac{d}{d x}\left(e^{i\left(t_{j}-x\right) A} f(x)\right) d x d w_{s}\right\|_{H}^{2} \\
& \leq \sum_{j=1}^{k} E\left(\int_{t_{j}-1}^{t_{j}} \int_{t_{j}-1}^{s}\left\|-i A e^{i\left(t_{j}-x\right)} A f(x)+e^{i\left(t_{j}-x\right)} A f^{\prime}(x)\right\|_{H} d x d w_{s}\right)^{2} \\
& \leq M_{4}^{2} \sum_{j=1}^{k} E\left(\int_{t_{j}-1}^{t_{j}} \int_{t_{j}-1}^{s} d x d w_{s}\right)^{2} \\
& \leq M_{4}^{2} \sum_{j=1}^{k} \int_{t_{j}-1}^{t_{j}}\left(\int_{t_{j}-1}^{s} d x\right)^{2} d s \leq M_{4}^{2} \sum_{j=1}^{k} \tau^{3} \leq M_{4}^{2} \tau .
\end{aligned}
$$

Let us estimate $T_{3 k}$. For $k \neq j$

$$
\begin{gathered}
E\left\|T_{3 k}\right\|_{H}^{2}=E\left\|-i \sum_{j=1}^{k} R^{k-j}\left[e^{i \tau A}-R\right] f\left(t_{j-1}\right) \Delta w_{j}\right\|^{2} \\
\leq \sum_{j=1}^{k}\left\|A R^{k-j}\right\|_{H}^{2}\left\|A^{-2}\left[e^{i \tau A}-R\right]\right\|_{H}^{2}\left\|A f\left(t_{j-1}\right)\right\|_{H}^{2} j \\
\leq \sum_{j=1}^{k} \frac{M_{1}^{2}}{j \tau^{2}} M_{2}^{2} \tau^{4} M_{4}^{2} j \leq M_{1}^{2} M_{2}^{2} M_{4}^{2} T \tau .
\end{gathered}
$$

For $k=j$, using the Taylor expansion formula for exponential function and $R$, easily seen that $\max _{0 \leq k \leq N}\left(E\left\|T_{3 k}\right\|_{H}^{2}\right)^{1 / 2} \leq M \tau^{1 / 2}$. Therefore,

$$
\max _{0 \leq k \leq N}\left(E\left\|T_{3 k}\right\|_{H}^{2}\right)^{1 / 2} \leq M \tau^{1 / 2} .
$$

Hence the result follows from the estimates of $T_{1 k}, T_{2 k}, T_{3 k}$. 


\section{Numerical Results}

In this section, the numerical experiments of the initial value problem

$$
\left\{\begin{array}{l}
i d u(t, x)-u_{x x}(t, x) d t=i e^{i t \pi^{2}} \sin (\pi x) d w_{t}, \\
0<t, x<1, \quad u(0, x)=0, \quad 0 \leq x \leq 1, \\
u(t, 0)=u(t, 1)=0, \quad 0 \leq t \leq 1,
\end{array}\right.
$$

for the stochastic Schrödinger equation using Rothe-Maruyama difference scheme are presented. It is clear that this problem satisfies the assumptions of Theorem 2. The exact solution of this problem is

$$
u(t, x)=e^{i t \pi^{2}}(\sin \pi x) w_{t} .
$$

Here $w_{t}=\sqrt{t} \xi, \xi \in N(0,1)$. For the approximate solution of problem (15), the set $[0,1]_{\tau} \times[0,1]_{h}$ of a family of grid points depending on the small parameters $\tau$ and $h$

$$
\begin{aligned}
{[0,1]_{\tau} \times[0,1]_{h} } & =\left\{\left(t_{k}, x_{n}\right): t_{k}=k \tau, 0 \leq k \leq N, \quad N \tau=1\right. \\
x_{n} & =n h, 0 \leq n \leq M, M h=1\}
\end{aligned}
$$

is defined. We suggest the following Rothe-Maruyama difference scheme for the approximate solution of problem (15)

$$
\left\{\begin{array}{l}
i\left(u_{n}^{k}-u_{n}^{k-1}\right)-\frac{\left(u_{n+1}^{k}-2 u_{n}^{k}+\pi^{2} u_{n-1}^{k}\right) \tau}{h^{2}}=f\left(t_{k}, x_{n}\right) \Delta w_{k}, \\
1 \leq k \leq N, \quad 1 \leq n \leq M-1, \quad \Delta w_{k}=w_{k}-w_{k-1}, \\
u_{n}^{0}=0,1 \leq n \leq M-1, \quad u_{0}^{k}=0, u_{M}^{k}=0,0 \leq k \leq N .
\end{array}\right.
$$

So we have $(N+1) \times(N+1)$ system of linear equations which can be written in the matrix form as:

$$
\left\{\begin{array}{l}
A U_{n+1}+B U_{n}+C U_{n-1}=D \varphi_{n}, \quad 1 \leq n \leq M-1 \\
U_{0}=0, U_{M}=0
\end{array}\right.
$$

where

$$
\varphi_{n}=\left[\begin{array}{c}
\varphi_{n}^{0} \\
\varphi_{n}^{1} \\
\cdots \\
\varphi_{n}^{N}
\end{array}\right]_{(N+1) \times 1}, \quad \varphi_{n}^{k}=\left\{\begin{aligned}
0, & k=0, \\
f\left(t_{k}, x_{n}\right), & 1 \leq k \leq N,
\end{aligned}\right.
$$


$A(i, i+1)=a, B(i, i+1)=c, C(i, i+1)=d$ for any $1 \leq i \leq N, B(i, i)=b$ for any $1 \leq i \leq N+1, B(N+1,1)=1$ and the other entries for the matrices $A$, $B$ and $C$ are all zero. The matrix $D$ is an identity matrix of order $N+1$ and

$$
U_{s}=\left[U_{s}^{0}, U_{s}^{1}, \ldots, U_{s}^{N-1}, U_{s}^{N}\right]^{t}, \quad s=n-1, n, n+1 .
$$

In the above matrices entries are

$$
a=-\frac{\tau}{h^{2}}, b=-i, c=i+\frac{2 \tau}{h^{2}}, d=-\frac{\tau}{h^{2}} .
$$

Thus, we have the first order difference equation with respect to $n$ with matrix coefficients. To solve this difference equation we have applied the same modified Gauss elimination method for the difference equation with respect to $n$ with matrix coefficients as in [7]. For the comparison of the numerical solution of the difference equation and the analytical solution of the differential equation, the error terms are computed by the following formulation:

$$
E_{M}^{N}=\max _{1 \leq k \leq N} \frac{1}{N_{\text {sim }}} \sum_{j=1}^{N_{\text {sim }}}\left(\sum_{n=1}^{M-1}\left[u\left(t_{k}, x_{n}\right)-u_{n}^{k}\right]^{2} h\right)^{1 / 2} .
$$

The numerical solutions of the problem (15) are recorded for various values of $N$ and $M$ based on the numerical scheme (16), where $u\left(t_{k}, x_{n}\right)$ represents the exact solution and $u_{n}^{k}$ represents the numerical solution at $\left(t_{k}, x_{n}\right)$. The result are shown in the Table 1 for $N=M=5,10,20,40$. In all of these numerical experiments the number of simulations $N_{\text {sim }}$ is kept constant at 1000 . Hence, each numerical problem has been solved based on 1000 different sample paths for the process of standard Brownian motion $w_{t}$.

Table 1. Comparison of the errors for the exact solution of the differential equation (15) and the numerical solution of the Rothe-Maruyama difference scheme (16).

\begin{tabular}{cccc}
\hline$N=M=5$ & $N=M=10$ & $N=M=20$ & $N=M=40$ \\
\hline 0.37923 & 0.2557 & 0.13629 & 0.06927 \\
\hline
\end{tabular}

From Table 1 it is seen that, using the Monte Carlo simulation, the RotheMaruyama difference (16) converges to the solution of stochastic Schrödinger equation (15). 


\section{Acknowledgement}

The author express his gratitude to Prof. Allaberen Ashyralyev, Prof. Charyyar Ashyralyyev and reviewers for their valuable comments.

\section{References}

[1] A. Ashyralyev, On modified Crank-Nicholson difference schemes for stochastic parabolic equation, Numer. Funct. Anal. Optim., 29, No 3-4 (2008), 268-282.

[2] A. Ashyralyev, A. Sirma, Nonlocal boundary value problems for the Schrödinger equation, Comput. Math. Appl., 55 (2008), 392-407.

[3] A. Kharab, H. Eleuch, Analytical solution of the position dependent mass Schrödinger equation with a hyperbolic tangent potential, International Journal of Applied Mathematics, 32, No 2 (2019), 357-367; doi:10.12732/ijam.v32i2.14.

[4] A. Ashyralyev, M.E. San, An approximation of semigroups method for stochastic parabolic equations, Abstr. Appl. Anal., 2012 (2012), 1-24; doi:10.1155/2012/684248.

[5] C. Privot, M. Röchner, A Concise Course on Stochastic Partial Differential Equations, Springer-Verlag, Berlin (2007).

[6] L. Bouten, M. Guta, H. Maassen, Stochastic Schrödinger equations, J. Phys. A. Math. Gen., 37 (2004), 3189-3209.

[7] C. Ashyralyyev, Stability of Rothe difference scheme for the reverse parabolic problem with integral boundary condition, Math. Meth. Appl. Sci., 43 (2020), 5369-5379.

[8] H.O. Fattorini, Second Order Linear Differential Equations in Banach Space, Notas de Matematica, North-Holland (1985).

[9] A. Ashyralyev, H.O. Fattorini, On uniform difference-schemes for 2ndorder singular pertubation problems in Banach spaces, SIAM J. Math. Anal., 23, No 1 (1992), 29-54.

[10] G. Da Prato, J. Zabczyk, Stochastic Equations in Infinite Dimensions, Encyclopedia of Mathematics and its Applications Book 152, Cambridge University Press (2014). 
[11] A. Ashyralyev, D. Agirseven, On the stable difference schemes for the Schrödinger equation with time delay, Computational Methods in Applied Mathematics, 20, No 1 (2020), 27-38. 